\title{
THE JOIN OF THE VARIETIES OF STRICT INVERSE SEMIGROUPS AND RECTANGULAR BANDS
}

\author{
by MARIO PETRICH and NORMAN R. REILLY $\dagger$
}

(Received 19 August, 1982)

1. Introduction and summary. In recent years, certain varieties of semigroups with unary operations (of "inversion") have received considerable attention. Generally speaking, these have been contained in one or other of the two classes of completely regular semigroups (that is, semigroups that are unions of groups) and inverse semigroups. For instances of the former see $[\mathbf{1}],[\mathbf{2}],[\mathbf{3}],[\mathbf{6}],[\mathbf{1 0}],[\mathbf{1 4}]$ and $[\mathbf{1 5}]$, and for instances of the latter see [7], [8], [12] and [13].

The varieties of all completely regular semigroups and of all inverse semigroups have relatively little in common, in that their intersection is simply the variety of all semilattices of groups. As a result, the investigations have been conducted independently with no interplay between the two theories.

The purpose of this note is to move towards viewing completely regular semigroups and inverse semigroups as members of the variety of unary semigroups and to study classes of unary semigroups with larger intersections with completely regular semigroups and inverse semigroups than merely semilattices of groups. As a first step in this programme, we consider here the join of the variety of rectangular bands and the variety of inverse semigroups generated by Brandt semigroups (that is, the variety of strict inverse semigroups) as varieties of unary semigroups. Equivalently (as we shall show) we consider the join of the variety of orthodox normal bands of groups and the variety of strict combinatorial inverse semigroups.

In this way we break out from the lattices of varieties of completely regular semigroups and of inverse semigroups and initiate the investigation of the joins of their subvarieties. This study opens up new vistas in the theory of varieties of unary semigroups.

The second section brings together important background information. In Section 3, we define the variety of strict *-semigroups $\mathscr{S}^{*}$, develop some of its elementary properties and give relevant examples. Section 4 introduces two important equivalence relations $\tau$ and $\tau^{\prime}$ on strict ${ }^{*}$-semigroups which are subsequently used to derive structurally important rectangular bands from any strict ${ }^{*}$-semigroup. The intersection of $\tau, \tau^{\prime}$ and the minimum inverse semigroup congruence $\gamma$ is also studied here.

In Section 5, it is shown that any strict *-semigroup is a subdirect product of rectangular bands, groups and 0 -direct products of rectangular bands with Brandt semigroups. From this representation theorem is derived the main result: $\mathscr{S}^{*}=\mathscr{R} \mathscr{B} \vee \mathscr{B} \vee \mathscr{G}$, where $\mathscr{R} \mathscr{B}, \mathscr{B}$ and $\mathscr{G}$ denote, respectively, the varieties of rectangular bands, combinatorial strict inverse semigroups and groups.

\footnotetext{
† This work was supported, in part, by NSERC grant A4044.
}

Glasgow Math. J. 25 (1984) 59-74. 
The lattice of subvarieties of $\mathscr{S}^{*}$ is considered in Section 6 and shown to be isomorphic to the direct product of the lattices of subvarieties of $\mathscr{R} \mathscr{B}, \mathscr{B}$ and $\mathscr{G}$.

A structure theorem is given for strict ${ }^{*}$-semigroups in Section 7 and the paper concludes in Section 8 with an example to show that, in general, the unary operation on a strict ${ }^{*}$-semigroup is not unique.

2. Background. Throughout we adopt the notation and terminology of Howie [5], to which the reader is referred for all undefined terms and notation. We adopt the following notation:

$\mathscr{T}$-the variety of all trivial semigroups,

$\mathscr{L} \mathscr{Z}$ - the variety of all left zero semigroups,

$\mathscr{R} \mathscr{L}$ - the variety of all right zero semigroups,

$\mathscr{R} \mathscr{B}$ - the variety of all rectangular bands,

$\mathscr{S}$-the variety of all semilattices,

$\mathscr{S} \mathcal{G}$-the variety of all semilattices of groups,

$\mathrm{B}_{2}$-the five element Brandt semigroup of rank 2,

$\mathscr{B}$ - the variety of all inverse semigroups generated by $B_{2}$, $\mathscr{L}(\mathcal{V})$-the lattice of all subvarieties of the variety $\mathcal{V}$.

Recall that a semigroup $S$ is said to be completely regular if it is a union of groups. The class $\mathscr{C} \mathscr{R}$ of completely regular semigroups is a variety of semigroups with unary operation defined by the identities

$$
x x^{-1} x=x, \quad\left(x^{-1}\right)^{-1}=x, \quad x x^{-1}=x^{-1} x .
$$

Various subvarieties of $\mathscr{C} \mathscr{R}$ have been extensively studied and certain parts of the lattice $\mathscr{L}(\mathscr{C} \mathscr{R})$ are well understood: see [3], [6], [10], [14] and [15].

On the other hand the variety of inverse semigroups $\mathscr{I}$ has also been the object of much interest. As semigroups with a unary operation $\Phi$ is defined by the identities

$$
x x^{-1} x=x, \quad\left(x^{-1}\right)^{-1}=x, \quad x x^{-1} y y^{-1}=y y^{-1} x x^{-1}, \quad(x y)^{-1}=y^{-1} x^{-1} .
$$

For further information see [7], [8], [12] and [13].

It is well known and easily seen that the intersection of these two varieties $\mathscr{C} \mathscr{R}$ and $\mathscr{I}$ is precisely the variety $\mathscr{S} \mathscr{G}$. Our objective is to find varieties of unary semigroups which overlap more extensively than $\mathscr{Y} \mathscr{G}$ with $\mathscr{C} \mathscr{R}$ and $\mathscr{\mathscr { T }}$. The most natural approach would appear to be to take small varieties from $\mathscr{L}(\mathscr{C} \mathscr{R})$ and $\mathscr{L}(\mathscr{F})$, such as $\mathscr{R} \mathscr{B}$ and $\mathscr{B}$, and to study their join as varieties of unary semigroups. This is basically our approach except that we consider $\mathscr{R} \mathscr{B} \vee \mathscr{B} \vee \mathscr{G}$, since it is equally amenable to our techniques.

It is well known that $\mathscr{L}(\mathscr{R} \mathscr{B})$ is the four element lattice consisting of $\mathscr{T}, \mathscr{L} \mathscr{L}, \mathscr{R} \mathscr{L}$ and $\mathscr{R} \mathscr{B}$ and that $\mathscr{L}(\mathscr{B})$ is the three element chain consisting of $\mathscr{T}, \mathscr{S}$ and $\mathscr{B}$.

Varieties of inverse semigroups generated by Brandt semigroups were examined in $[\mathbf{8}]$ and $[\mathbf{1 2}]$, from which the next three lemmas are drawn. 
LEMMA 2.1. Let $\mathcal{V}$ be a variety of inverse semigroups which is not a variety of groups. Then $\mathscr{S} \subseteq \mathscr{V}$. Moreover, the following are equivalent:

(i) every element of $\mathcal{V}$ is a group or a semilattice of groups,

(ii) the identity $x x^{-1}=x^{-1} x$ holds in $\mathscr{V}$,

(iii) $\mathscr{V}=\mathscr{S} \vee \mathcal{U}$, where $\mathcal{U}$ is the variety of groups $\mathscr{V} \cap \mathscr{G}$,

(iv) $\mathscr{S} \subseteq \mathscr{V} \subseteq \mathscr{S} \mathscr{G}$.

Since the variety $\mathscr{B}$ has an important role in our discussions, we provide some alternative characterizations of it.

DefinITION 2.2. A semigroup is said to be combinatorial if it contains no non-trivial subgroups.

LEMMA 2.3. For an inverse semigroup $S$, the following are equivalent:

(i) $S \in \mathscr{B}$,

(ii) $S$ satisfies the identity $\left(x y x^{-1}\right)^{2}=x y x^{-1}$,

(iii) $S$ is a subdirect product of combinatorial Brandt semigroups or is a one element group.

We shall also require some information regarding varieties generated by more general Brandt semigroups.

LEMMA 2.4. Let $\mathcal{V}$ be a variety of inverse semigroups which is not a variety of semilattices of groups. Then $\mathscr{B} \subseteq \mathscr{V}$. Moreover, the following are equivalent:

(i) every member of $\mathcal{V}$ is a subdirect product of groups and/or Brandt semigroups,

(ii) the identity $\left(x y x^{-1}\right)\left(x y x^{-1}\right)^{-1}=\left(x y x^{-1}\right)^{-1}\left(x y x^{-1}\right)$ holds in $\mathscr{V}$,

(iii) $\mathcal{V}=\mathscr{B} \vee \mathcal{U}$, where $\mathcal{U}$ is the variety of groups $\mathcal{V} \cap \mathscr{G}$,

(iv) $\mathscr{B} \subseteq \mathscr{V} \subseteq \mathscr{B} \vee \mathscr{G}$.

Definition 2.5. An inverse semigroup $S$ is said to be strict if it is a subdirect product of groups and/or Brandt semigroups, that is, if and only if $S \in \mathscr{B} \vee \mathscr{G}$.

The elements of $\mathscr{B}$ could be characterized now as combinatorial strict inverse semigroups.

DefinItION 2.6 [11]. A semigroup $S$ is said to satisfy $\mathscr{D}$-majorization if, for any $e \in E_{\mathrm{S}}$ such that there exists an idempotent $f \in D_{a}$ with $f \leq e$, there is a maximum such idempotent.

The importance here of the concept of $\mathscr{D}$-majorization lies in the next two results due to Lallement [9] (see also [11]).

LeMmA 2.7. Let $D_{a}, D_{b}(a, b \in S)$ be $\mathscr{D}$-classes of a completely semisimple semigroup $S$. Let $f \in D_{a}$ be an idempotent such that there exists exactly one idempotent $g \in D_{b}$ with $g \leq f$. Then for every idempotent $e \in D_{a}$ there is a unique idempotent $\bar{e} \in D_{b}$ with $\bar{e} \leq e$. Let the function $\varphi_{a, b}: D_{a} \rightarrow D_{b}$ be defined by

$$
\varphi_{a, b}: x \rightarrow \bar{e} x=x \bar{f} \quad\left(x \in D_{a}\right)
$$


where $e \mathscr{R} x$ and $x \mathscr{L} f$. Then $\varphi_{a, b}$ is well defined and, for any $x \in D_{a}, y \in D_{b}$,

(i) $x y \in D_{b} \Rightarrow x y=\left(x \varphi_{a . b}\right) y, \quad y x \in D_{b} \Rightarrow y x=y\left(x \varphi_{a . b}\right)$,

(ii) If $D_{c} \leq D_{b} \leq D_{a}$, then $\varphi_{a, b} \varphi_{b, c}=\varphi_{a, c}$.

Furthermore, the mapping $\varphi_{b}: S \rightarrow J(b) / I(b)$ defined by

$$
x \varphi_{b}= \begin{cases}x \varphi_{a, b} & \text { if } \quad J(b) \subseteq J(a) \\ 0 & \text { otherwise }\end{cases}
$$

is a homomorphism which is one-to-one on $J_{b}$.

It should be noted that, in Lemma 2.7 , if $a \mathscr{D} c$ and $b \mathscr{D} d$, then $\varphi_{a, b}=\varphi_{c, d}$. The first condition in the next theorem provides the most convenient test for $\mathscr{D}$-majorization.

THEOREM 2.8. The following two conditions on a regular semigroup $S$ are equivalent:

(i) for any $e, f, g \in E_{S}$, if $e \geq f, e \geq g$ and $f \mathscr{D} g$ then $f=g$,

(ii) $S$ is completely semisimple and satisfies $\mathscr{D}$-majorization.

Whenever (i) and (ii) hold, the mapping $x$ of $S$ into the product $\prod_{J(a)} J(a) / I(a)$ of the principal factors of $S$ defined by

$$
\chi: x \rightarrow \chi_{x} \text { where } \chi_{x}(J(a))=x \varphi_{a}
$$

embeds $S$ as a subdirect product.

Notation 2.9. We shall consistently use the notation $\varphi_{a, b}, \varphi_{a}$ and $\chi$ to denote the mappings introduced in Lemma 2.7 and Theorem 2.8. In addition, since the mappings $\varphi_{a . b}$ and $\varphi_{a}$ depend only on the $\mathscr{D}$-classes of $a$ and $b$, we shall also write $\varphi_{\alpha, \beta}=\varphi_{a, b}=\varphi_{c, d}$ and $\varphi_{a}=\varphi_{\alpha}=\varphi_{c}$ whenever $a \mathscr{D} c, b \mathscr{D} d, \alpha=D_{a}$ and $\beta=D_{b}$.

3. Strict *-semigroups. We introduce here the semigroups in the title and establish some of their properties which will be needed later.

Defintrion 3.1. A semigroup $S$ with a unary operation * is a strict ${ }^{*}$-semigroup if it satisfies the following identities:

(I) $x=x x^{*} x$,

(II) $x=\left(x^{*}\right)^{*}$,

(III) $x x^{*} x^{*} x=x^{*} x x x^{*}$,

(IV) $\left(x y x^{*}\right)\left(x y x^{*}\right)^{*}=\left(x y x^{*}\right)^{*}\left(x y x^{*}\right)$,

(V) $x(y z)^{*} w=x z^{*} y^{*} w$

(VI) $(x y)^{*}=\left(x^{*} x y\right)^{*}\left(x y y^{*}\right)^{*}$.

We denote by $\mathscr{S}^{*}$ the variety of all strict *-semigroups.

Note. The above identities do not include and do not imply the identity

$$
(x y)^{*}=y^{*} x^{*} \text {. }
$$

This identity together with (I) and (II) defines the class of regular *-semigroups. Consequently the semigroups considered here are not, in general, regular *-semigroups. 
Several of the identities (I)-(VI) are modelled on identities that figure prominently in the studies of varieties of completely regular or inverse semigroups. For instance, (I) and (II) hold in both completely regular and inverse semigroups, with (I) implying regularity. Identity (III) is a weakened form of commutativity of idempotents in inverse semigroups as well as the identity $x x^{-1}=x^{-1} x$ in completely regular semigroups, Identity (IV) is important in the study of varieties of inverse semigroups (see Lemma 2.4) while identities (V) and (VI) are weakened forms of the inverse of a product rule $(x y)^{-1}=y^{-1} x^{-1}$ in inverse semigroups.

We now develop some basic properties of strict *-semigroups.

LEMMA 3.2. If $e$ is an idempotent of a strict ${ }^{*}$-semigroup, then $e^{*}=e$.

Proof. Indeed, using identities (I), (II) and (III), we obtain

$$
e^{*}=e^{*} e e^{*}=e^{*} e e e^{*}=e e^{*} e^{*} e=e\left(e e^{*} e^{*} e\right) e=e\left(e^{*} e e e^{*}\right) e=\left(e e^{*} e\right)^{2}=e^{2}=e .
$$

Note. A semigroup $S$ is a generalized inverse semigroup (Yamada [16]) if it is orthodox and the idempotents of $S$ form a normal band. It follows immediately from Lemma 3.2 and identity (V) that any strict *-semigroup is a generalized inverse semigroup.

LEMMA 3.3. Every strict ${ }^{*}$-semigroup $S$ is orthodox and satisfies the identities:

(VII) $(x y x)^{*}=x^{*} y^{*} x^{*}$,

(VIII) $x y y^{*} z z^{*} w=x z z^{*} y y^{*} w$

(IX) $(x y)(x y)^{*}=\left(x y y^{*}\right)\left(x y y^{*}\right)^{*}$,

(X) $(x y)^{*}(x y)=\left(x^{*} x y\right)^{*}\left(x^{*} x y\right)$.

Proof. Let $e, f \in E_{\mathrm{S}}$. Using identities (I) and (V) and Lemma 3.2, we get

$$
e f=e f(e f)^{*} e f=e f f^{*} e^{*} e f=e f e f
$$

and $S$ is orthodox.

Let $x, y \in S$. Using identities (VI), (I) and (IV), we obtain

$$
\begin{aligned}
(x y x)^{*} & =[(x y) x]^{*}=\left[(x y)^{*} x y x\right]^{*}\left(x y x x^{*}\right)^{*} \\
& =\left[(x y)^{*} x y x\right]^{*}\left(x y x x^{*}\right)^{*}\left(x y x x^{*}\right)\left(x y x x^{*}\right)^{*} \\
& =\left[(x y)^{*} x y x\right]^{*}\left[\left(x y x x^{*}\right)^{*}\right]^{2}\left(x y x x^{*}\right),
\end{aligned}
$$

whence $(x y x)^{*}=(x y x)^{*} x x^{*}$; symmetrically $(x y x)^{*}=x^{*} x(x y x)^{*}$.

We now use identities (V) and (I) to obtain

$$
(x y x)^{*}=x^{*} x(x y x)^{*} x x^{*}=x^{*} x\left(x^{*} y^{*} x^{*}\right) x x^{*}=x^{*} y^{*} x^{*} .
$$

Hence $S$ satisfies identity (VII).

Since $e f \in E_{S}$, by Lemma 3.2 we obtain, for any $x, w \in S$,

$$
x e f w=x(e f)^{*} w=x f^{*} e^{*} w=x f e w,
$$

which proves identity (VIII). For any $x, y \in S$, using identities (VII), (V), (VIII) and (I), we 
obtain

$$
\begin{aligned}
(x y)(x \dot{y})^{*} & =x y\left(x^{*} x y\right)^{*}\left(x y y^{*}\right)^{*} \\
& =x y y^{*} x^{*} x\left(x y y^{*}\right)^{*} \\
& =x x^{*} x y y^{*}\left(x y y^{*}\right)^{*} \\
& =\left(x y y^{*}\right)\left(x y y^{*}\right)^{*}
\end{aligned}
$$

giving identity (IX). The argument for identity $(\mathrm{X})$ is similar.

LEMMA 3.4. In a strict *-semigroup $S$, we have

(i) $a \mathscr{L} b \Leftrightarrow a=a b^{*} b$ and $b=b a^{*} a$,

(ii) $a \mathscr{R} b \Leftrightarrow a=b b^{*} a$ and $b=a a^{*} b$.

Proof. If $a \mathscr{L} b$, then $a=u b$ for some $u \in S$, so that $a=u b=u b b^{*} b=a b^{*} b$. The rest follows just as easily.

COROLlaRY 3.5. In a strict ${ }^{*}$-semigroup,

(i) $a \mathscr{H} a^{2} \Leftrightarrow a=a^{*} a^{2}=a^{2} a^{*}$

(ii) $a \mathscr{H} a^{2} \Rightarrow a^{*}=a^{-1}$, the inverse of $a$ in the group $H_{a}$.

Proof. (i) By Lemma 3.4 and identities (V), (III) and (I), we obtain

$$
\begin{aligned}
a \mathscr{H} a^{2} & \Leftrightarrow a=a\left(a^{2}\right)^{*} a^{2}=a^{2}\left(a^{2}\right)^{*} a \text { and } a^{2}=a^{2} a^{*} a=a a^{*} a^{2} \\
& \Leftrightarrow a=a a^{*} a^{*} a a=a a a^{*} a^{*} a \\
& \Leftrightarrow a=a^{*} a a a^{*} a=a a^{*} a a a^{*} \\
& \Leftrightarrow a=a^{*} a^{2}=a^{2} a^{*} .
\end{aligned}
$$

(ii) Of course, $a \mathscr{H} a^{2}$ is equivalent to saying that $H_{a}$ is a subgroup of $S$ and then, by (i), $a a^{-1} a^{*}=a^{*} a a a^{-1} a^{*}=a^{*} a a^{*}=a^{*}$ and similarly $a^{*}=a^{*} a^{-1} a$. From this and (i) it follows that $a^{*} \mathscr{H} a$ so that, by (I), $a^{*}=a^{-1}$.

COROllary 3.6. In a strict *-semigroup $S$, we have $a b a^{*} \mathscr{H}\left(a b a^{*}\right)^{2}$, for all $a \in S$.

Proof. From (IV), with $u=a b a^{*}$, we have $u u^{*}=u^{*} u$ and so, multiplying on the right by $u, u=u^{*} u^{2}$. By Corollary $3.5(i)$, this implies that $u \mathscr{H} u^{2}$.

LEMMA 3.7. Every strict *-semigroup $S$ is completely semisimple and satisfies $\mathscr{D}$ majorization.

Proof. We verify condition (i) of Theorem 2.8. Let $e, f, g \in E_{\mathrm{S}}$ be such that $e \geq f, e \geq \mathrm{g}$ and $f \mathscr{D} g$. Then $f \mathscr{L} a$ and $a \mathscr{R} g$, for some $a \in S$, so that $f=b a, a=c f, g=a d, a=g t$, for some $b, c, d, t \in S$ and $a=a f=g a$. Thus $a=a e=e a$, whence $a=e a e \mathscr{H}(e a e)^{2}=a^{2}$ by Lemma 3.2 and Corollary 3.6. Therefore $a$ is contained in a maximal subgroup of $S$ with identity $u$, say. Then

$$
f=b a=b a u=f u=e f u e=e u f e=u f=u
$$

and analogously $g=u$, which proves that $f=g$, as required. 
It follows from Lemma 3.7 that $\mathscr{D}=\mathscr{J}$ in any strict *-semigroup and that we may consider the set of $\mathscr{D}$-classes ( $\mathscr{F}$-classes) as a partially ordered set.

LEMmA 3.8. Let $S$ be a strict ${ }^{*}$-semigroup. In the notation of Lemma 2.7, if $D_{b} \leq D_{a}$ and $x \in D_{a}$ then $x^{*} \varphi_{a, b}=\left(x \varphi_{a, b}\right)^{*}$.

Proof. If $e=x x^{*}$, then $e \mathscr{R} x$ and $e \mathscr{L} x^{*}$. Let $\bar{e}$ be the unique idempotent in $D_{\mathrm{b}}$ with $\bar{e} \leq e$. Then $x \varphi_{a, b}=\bar{e} x$ and $x^{*} \varphi_{a, b}=x^{*} \bar{e}$ so that, by (V), (VII) and Lemma 3.2, we have

$$
\left(x \varphi_{a, b}\right)^{*}=(\bar{e} x)^{*}=\left(x x^{*} \bar{e} x\right)^{*}=x^{*}\left(x^{*} \bar{e}\right)^{*} x^{*}=x^{*} \bar{e} x x^{*}=x^{*} \bar{e}=x^{*} \varphi_{a, b}
$$

The following is a simple but important consequence of these preliminary observations.

Propostrion 3.9. Every strict ${ }^{*}$-semigroup is a subdirect product of completely 0 -simple and/or completely simple strict *-semigroups.

Proof. This follows immediately from Lemmas 3.7 and 3.8 and Theorem 2.8.

Some elementary examples of strict *-semigroups are the following.

(i) Groups: the *-operation is just the usual inverse operation.

(ii) Rectangular bands: the *-operation is just the identity operation.

(iii) Rectangular groups: the *-operation gives the inverse within each subgroup.

(iv) Brandt semigroups: the *-operation is the usual inverse operation.

In all of these cases, the *-operation is unique.

The class of examples that we are about to describe is central to the whole discussion. We first require a certain construction.

Definition 3.10 [4]. Let $A$ and $B$ be semigroups and let $B$ have a zero. Let $I=\left\{(a, 0)\{a \in A\}\right.$. Then $I$ is an ideal of $A \times B$ and the 0 -direct product $A \times{ }_{0} B$ of $A$ and $B$ is the Rees factor semigroup $(A \times B) / I$. Strictly speaking $I$ is now the zero of $A \times{ }_{0} B$ but it is notationally convenient to identify any element of the form $(a, 0)$ with $I$ and to denote this element by 0 .

Note that any variety is closed under the formation of 0 -direct products.

LEMMA 3.11. Let $A$ be a rectangular band and $B$ be a Brandt semigroup. Then $A \times{ }_{0} B$ is a strict *-semigroup where $(a, b)^{*}=\left(a, b^{-1}\right)$.

Proof. Straightforward.

The *-operation in the class of strict *-semigroups introduced in Lemma 3.11 is not, in general, the only unary operation satisfying the identities (I)-(VI). An example to illustrate this will be considered in Section 8 .

Whenever a semigroup $A \times_{0} B$ as in Lemma 3.11 is being considered as a strict *-semigroup, we shall mean this with respect to the unary operation in Lemma 3.11.

4. Some basic equivalence relations. In this section we introduce two equivalence relations on strict ${ }^{*}$-semigroups and investigate their basic properties, particularly in 
relation to the minimum inverse semigroup congruence. This provides the foundations for the representations developed later.

Lemma 4.1. Let $S$ be a strict ${ }^{*}$-semigroup. Define the relations $\tau=\tau_{\mathrm{S}}$ and $\tau^{\prime}=\tau_{\mathrm{S}}^{\prime}$ on $S$ by

$$
\begin{gathered}
x \tau y \Leftrightarrow \text { there exists } z \in S \text { with } x \mathscr{R} z \text { and } z^{*} \mathscr{R} y, \\
x \tau^{\prime} y \Leftrightarrow \text { there exists } z \in S \text { with } x \mathscr{L} z \text { and } z^{*} \mathscr{L} y .
\end{gathered}
$$

Then $\tau$ and $\tau^{\prime}$ are equivalence relations on $S$ with the following properties:

(i) $\mathscr{R} \subseteq \tau \subseteq \mathscr{D}, \quad \mathscr{L} \subseteq \tau^{\prime} \subseteq \mathscr{D}$;

(ii) $x\left(\tau \cap \tau^{\prime}\right) x^{*}$, for all $x \in S$.

Proof. By duality, it suffices to consider $\tau$. Clearly $\tau \subseteq \mathscr{D}$, so let $x, y, z$ be elements of a single $\mathscr{D}$-class $D$. Then $x \mathscr{R} x x^{*}$ and $\left(x x^{*}\right)^{*}=x x^{*} \mathscr{R} x$ so that $x \tau x$ and $\tau$ is reflexive. If $x \tau y$ then, for some $u \in S, x \mathscr{R} u$ and $u^{*} \mathscr{R} y$. Hence $y \mathscr{R} u^{*}$ and $\left(u^{*}\right)^{*}=u \mathscr{R} x$. Thus y $\tau x$ and $\tau$ is symmetric. If $x \tau y$ and $y \tau z$, then there exist $u, v \in S$ with $x \mathscr{R} u, u^{*} \mathscr{R} y, y \mathscr{R} v$, $v^{*} \mathscr{R} z$. It follows that $u^{*} \mathscr{R} v$ and $u^{*} u v=v \in D$, so that $u v \in D$. Since $S$ is completely semisimple, this implies that $u \mathscr{R} u v$ so that $x \mathscr{R} u \mathscr{R} u v$. Using identity $(X)$, we get

$$
(u v)^{*}(u v)=\left(u^{*} u v\right)^{*}\left(u^{*} u v\right)=v^{*} v
$$

whence $(u v)^{*} \mathscr{R} v^{*} \mathscr{R} z$ and $x \tau z$. Thus $\tau$ is transitive.

If $x \mathscr{R} y$, then $x \mathscr{R} x x^{*} \mathscr{R} y$ so that $x \mathscr{R} x x^{*}$ and $\left(x x^{*}\right)^{*}=x x^{*} \mathscr{R} y$, by Lemma 3.2 ; thus $x \tau y$ and (i) holds for $\tau$. Finally, $x \mathscr{R} x$ and $x^{*} \mathscr{R} x^{*}$ so that $x \tau x^{*}$. This completes the proof of the lemma.

LEMmA 4.2. Let $S$ and $T$ be unary semigroups satisfying (I), (II) and (III). Let $T$ be such that there exists only one unary operation satisfying (I), (III) and $x^{*}=x^{*} x x^{*}$. Let $\theta: S \rightarrow T$ be a semigroup homomorphism. Then $\theta$ is also a ${ }^{*}$-semigroup homomorphism.

Proof. For any $x \in S$, we have

$$
\begin{aligned}
& x \theta=\left(x x^{*} x\right) \theta=(x \theta)\left(x^{*} \theta\right)(x \theta), \quad x^{*} \theta=\left(x^{*} x x^{*}\right) \theta=\left(x^{*} \theta\right)(x \theta)\left(x^{*} \theta\right), \\
& (x \theta)\left(x^{*} \theta\right)\left(x^{*} \theta\right)(x \theta)=\left(x x^{*} x^{*} x\right) \theta=\left(x^{*} x x x^{*}\right) \theta=\left(x^{*} \theta\right)(x \theta)(x \theta)\left(x^{*} \theta\right) .
\end{aligned}
$$

By the uniqueness of ${ }^{*}$ in $T$, we have $x^{*} \theta=(x \theta)^{*}$.

COROLlary 4.3. Let $S$ by a unary semigroup satisfying (I), (II) and (III) and let $\theta: S \rightarrow T$ be a semigroup homomorphism. If $T$ is an inverse semigroup or a completely regular semigroup, then $\theta$ is also $a .^{*}$-semigroup homomorphism.

Recall [5] that on any orthodox semigroup there is a minimum congruence such that the quotient is an inverse semigroup. To describe this, we require the following notation.

Notation 4.4. For any element $x$ in a semigroup $S$, let

$$
V(x)=\{y \in S \mid x y x=x \text { and } y x y=y\}
$$

that is, let $V(x)$ denote the set of inverses of $x$. Also let $\gamma$ denote the relation defined on $S$ 
by

$$
x \gamma y \Leftrightarrow V(x)=V(y) .
$$

Note that in a strict ${ }^{*}$-semigroup we always have $x^{*} \in V(x)$.

LEMMA 4.5 [5]. Let $S$ be an orthodox semigroup. Then $\gamma$ is the smallest congruence on $S$ such that $S / \gamma$ is an inverse semigroup. Moreover, $\gamma \subseteq \mathscr{D}$ and $\gamma \cap \mathscr{H}$ is the identity relation on S.

LEMMA 4.6. Let $S$ be a strict *-semigroup. Then $\tau \cap \tau^{\prime} \cap \gamma$ is the identity relation on $S$.

Proof. Let $(x, y) \in \tau \cap \tau^{\prime} \cap \gamma$. Since $(x, y) \in \tau$, we have $x \mathscr{D} y$ and there exists an element $z$ with $x \mathscr{R} z$ and $z^{*} \mathscr{R} y$. Then, by (V),

$$
z^{2}=z z^{*} z z=z z^{*}\left(z^{*}\right)^{*} z=z z^{*}\left(y y^{*} z^{*}\right)^{*} z=z z^{*} z\left(y y^{*}\right)^{*} z=z y y^{*} z=z y y^{*} x x^{*} z .
$$

Since $S / \gamma$ is an inverse semigroup and $x \gamma=y \gamma$, it follows from Corollary 4.3 that

$$
\begin{aligned}
\left(y y^{*} x x^{*}\right) \gamma & =(y \gamma)\left(y^{*} \gamma\right)(x \gamma)\left(x^{*} \gamma\right)=(y \gamma)(y \gamma)^{-1}(x \gamma)(x \gamma)^{-1} \\
& =(y \gamma)(y \gamma)^{-1}(y \gamma)(y \gamma)^{-1}=(y \gamma)(y \gamma)^{-1}=\left(y y^{*}\right) \gamma
\end{aligned}
$$

But $\gamma \subseteq \mathscr{D}$ and so $y y^{*} x x^{*} \mathscr{D} y y^{*} \mathscr{D} z$. Now $y=z^{*} z y$ and $z=x x^{*} z$ so that $z y, y y^{*} x x^{*}$ and $x^{*} z$ are all $\mathscr{D}$-equivalent (to $z$ ). Since $S$ is completely semisimple, it follows that $z^{2}=z y y^{*} x x^{*} z$ is also $\mathscr{D}$-equivalent to $z$ and therefore $z \mathscr{H} z^{2}$. By Corollary 3.5(ii), $z \mathscr{H} z^{*}$ from which we obtain $x \mathscr{R} z \mathscr{H} z * \mathscr{R} y$. Thus $x \mathscr{R} y$. Similarly, $(x, y) \in \tau^{\prime} \cap \gamma$ implies that $x \mathscr{L}$. Hence $\tau \cap \tau^{\prime} \cap \gamma \subseteq \mathscr{H}$. But, by Lemma $4.5, \gamma \cap \mathscr{H}$ is the identity relation and so the result follows.

LEMMA 4.7. Let $S$ be a strict *-semigroup and $a, b, c$ be $\mathscr{D}$-equivalent elements of $S$. Then there exists an element $z \in S$ with $z \tau=a \tau, z \tau^{\prime}=b \tau^{\prime}$ and $z \gamma=c \gamma$.

Proof. We first show that $a \tau \cap c \gamma \neq \varnothing$. Let $e=a a^{*}, f=c c^{*}$ and $x \in S$ be such that $e \mathscr{R} x$ and $x \mathscr{L} f$. Then $x f=x$ and, for some $x^{\prime} \in S, x^{\prime} x=f$. Let $x^{\prime \prime}=f x^{\prime}$. It is routine to verify that $x^{\prime \prime} \in V(x)$ and that $x^{\prime \prime} x=f$. Since $x^{\prime \prime} \in V(x), x^{\prime \prime} \gamma=(x \gamma)^{-1}=x^{*} \gamma$, so that

$$
\left(x^{*} x c\right) \gamma=\left(x^{\prime \prime} x c\right) \gamma=(f c) \gamma=c \gamma
$$

while $x^{*} x c \mathscr{R} x^{*}$ and $a \mathscr{R} x$. Thus $x^{*} x c \in a \tau \cap c \gamma$.

Similarly, $b \tau^{\prime} \cap c \gamma \neq \varnothing$. So let $u \in a \tau \cap c \gamma$ and $v \in b \tau^{\prime} \cap c \gamma$. Then

$$
\left(u u^{*}\right) \gamma=\left(c c^{*}\right) \gamma=\left(v v^{*}\right) \gamma=\left(u u^{*} v v^{*}\right) \gamma
$$

Hence

$$
u u^{*} v \mathscr{D} u u^{*} v v^{*} \gamma u u^{*} \mathscr{D} u \gamma c \gamma v
$$

and, since $\gamma \subseteq \mathscr{D}$, we have that $u u^{*} v, u$ and $v$ are $\mathscr{D}$-equivalent elements. Since $S$ is completely semisimple, this implies that $u u^{*} v \mathscr{R} u$ and $u u^{*} v \mathscr{L} v$ so that $u u^{*} v \in u \tau \cap v \tau^{\prime}=$ $a \tau \cap b \tau^{\prime}$, by Lemma 4.1(i), while $\left(u u^{*} v\right) \gamma=v \gamma=c \gamma$. Therefore $u u^{*} v \in a \tau \cap b \tau^{\prime} \cap c \gamma$. 
5. The variety of strict *-semigroups. We now wish to establish that the variety of strict ${ }^{*}$-semigroups is the join of the varieties of rectangular bands, of combinatorial strict inverse semigroups and of groups. To do so we develop a representation of strict *-semigroups as subdirect products of rectangular groups and 0-direct products of rectangular bands with Brandt semigroups.

Proposition 5.1. Let $S$ be a completely 0 -simple strict *-semigroup. Let I (respectively, $\Lambda$ ) denote the set of non-zero $\tau$ (respectively, $\tau^{\prime}$ ) classes of $S$. Consider $I$ (respectively, $\Lambda$ ) as a left zero (respectively, right zero) semigroup. Let $B=S / \gamma$. Then the mapping $\chi: S \rightarrow R=$ $(I \times \Lambda) \times_{0} B$ defined by

$$
x \chi= \begin{cases}\left(\left(x \tau, x \tau^{\prime}\right), x \gamma\right) & \text { if } x \neq 0 \\ 0 & \text { otherwise }\end{cases}
$$

is $a^{*}$-isomorphism.

Proof. By Lemma 4.6, $\tau \cap \tau^{\prime} \cap \gamma$ is the identity relation on $S$ and so it is clear that $\chi$ is one-to-one. From Lemma 4.7, it follows easily that $\chi$ maps $S$ onto $R$.

For $x, y \in S$, we have

$$
\begin{aligned}
(x \chi)(y \chi) & =\left(\left(x \tau, x \tau^{\prime}\right), x \gamma\right)\left(\left(y \tau, y \tau^{\prime}\right), y \gamma\right) \\
& =\left(\left(x \tau, y \tau^{\prime}\right),(x \gamma)(y \gamma)\right)=\left(\left(x \tau, y \tau^{\prime}\right),(x y) \gamma\right)
\end{aligned}
$$

while

$$
\left.(x y) \chi=\left((x y) \tau,(x y) \tau^{\prime}\right),(x y) \gamma\right) .
$$

If $x y=0$, then $(x y) \gamma=0$ and so the elements in (1) and (2) are both zero. If $x y \neq 0$, then $x \mathscr{R} x y$, so that $x \tau x y$, and $y \mathscr{L} x y$, so that $y \tau^{\prime} x y$. Thus the elements in (1) and (2) are again equal and $x$ is a homomorphism.

Furthermore, using Lemma 4.1(ii) and Corollary 4.3, we get

$$
\begin{aligned}
x^{*} \chi & =\left(\left(x^{*} \tau, x^{*} \tau^{\prime}\right), x^{*} \gamma\right)=\left(\left(x \tau, x \tau^{\prime}\right),(x \gamma)^{-1}\right) \\
& =\left(\left(x \tau, x \tau^{\prime}\right), x \gamma\right)^{*}=(x \chi)^{*}
\end{aligned}
$$

so that $\chi$ is a ${ }^{*}$-homomorphism and therefore a ${ }^{*}$-isomorphism.

Forgetting the unary operation for the moment, the semigroup $(I \times \Lambda) \times_{0} B$ of Proposition 5.1 is a special case of a construction introduced by Yamada [17] to study what he called generalized Brandt semigroups. Proposition 5.1 tells us, in effect, that completely 0-simple strict *-semigroups are, as semigroups, generalized Brandt semigroups. The converse does not hold.

Proposition 5.2. A completely simple strict *-semigroup is a rectangular group and conversely.

Proof. The direct part follows immediately from the fact that a strict *-semigroup is orthodox (Lemma 3.3) while the converse is obvious. 
Combining Propositions 3.9, 5.1 and 5.2, we arrive at the following subdirect product representation for strict ${ }^{*}$-semigroups.

THEOREM 5.3. Every strict *-semigroup is a subdirect product of rectangular bands, groups and/or 0-direct products of rectangular bands with Brandt semigroups.

We are now ready for the main result of this section.

THEOREM 5.4. $\mathscr{S}^{*}=\mathscr{R} \mathscr{B} \vee \mathscr{B} \vee \mathscr{G}$.

Proof. We have seen that every rectangular band, every Brandt semigroup and every group is a strict ${ }^{*}$-semigroup. Thus $\mathscr{R} \mathscr{B} \vee \mathscr{B} \vee \mathscr{G} \subseteq \mathscr{S}^{*}$. Now let $S \in \mathscr{S}^{*}$. By Theorem 5.3, $S$ is a subdirect product of rectangular bands, groups and/or 0-direct products of rectangular bands and Brandt semigroups. All rectangular bands and all groups lie in $\mathscr{R} \mathscr{B} \vee \mathscr{B} \vee \mathscr{G}$. So let $A \times{ }_{0} B$ be a 0 -direct product of a rectangular band $A$ and a Brandt semigroup $B$. By Lemma 2.4, $B \in \mathscr{B} \vee \mathscr{G}$. Hence $A \times B \in \mathscr{R} \mathscr{B} \vee \mathscr{B} \vee \mathscr{G}$ and so $A \times{ }_{0} B \in \mathscr{R} \mathscr{B} \vee \mathscr{B} \vee \mathscr{G}$. Consequently, $\mathscr{S}^{*} \subseteq \mathscr{R} \mathscr{B} \vee \mathscr{B} \vee \mathscr{G}$ and the result follows.

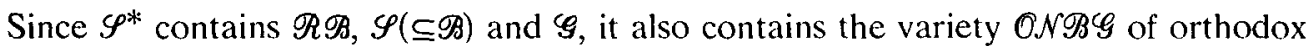
normal bands of groups (see [10] for details). Thus $O \mathcal{N} \mathscr{B} \mathscr{G} \wedge \mathscr{B} \subseteq \mathscr{G}^{*}$. Conversely, $O \mathcal{N} \mathscr{B} \mathscr{G} \wedge \mathscr{B}$ contains $\mathscr{R} \mathscr{B}, \mathscr{B}$ and $\mathscr{G}$ and therefore $\mathscr{S}^{*}$ so that we obtain an alternative characterization of the variety $\mathscr{S}^{*}$.

COROllary 5.5. $\mathscr{S}^{*}=\mathscr{O} \mathcal{N} \mathscr{B S} \vee \mathscr{B}$.

6. The lattice of subvarieties of $\mathscr{S}^{*}$. In this section we describe completely the lattice of subvarieties of $\mathscr{S}^{*}$ modulo group varieties.

THEOREM 6.1. The mapping

$$
\psi: \mathcal{U} \rightarrow(\mathcal{U} \cap \mathscr{R \mathscr { B }}, \mathcal{U} \cap \mathscr{B}, \mathcal{U} \cap \mathscr{G})
$$

is an isomorphism of $\mathscr{L}\left(\mathscr{S}^{*}\right)$ onto the lattice $\mathscr{L}(\mathscr{R} \mathscr{B}) \times \mathscr{L}(\mathscr{B}) \times \mathscr{L}(\mathscr{G})$.

Proof. Clearly $\psi$ is order preserving. Let $\boldsymbol{U}, \mathcal{V} \in \mathscr{L}\left(\mathscr{S}^{*}\right)$ be such that $\mathscr{U} \psi \leq \mathscr{V} \psi$ and let $S \in \mathcal{U}$. By Theorem 5.3, $S$ is a subdirect product of rectangular bands $R_{\alpha}(\alpha \in A)$, groups $G_{\beta}(\beta \in B)$ and/or 0-direct products of rectangular bands $R_{\gamma}(\gamma \in C)$ with Brandt semigroups $B_{\gamma}(\gamma \in C)$. Since $\mathscr{\psi} \leq \mathscr{V} \psi$, we see immediately that the $R_{\alpha}, G_{\beta}$ and $R_{\gamma}$ lie in $\mathscr{V}$. Let $B_{\gamma}=\mathscr{M}^{0}(I, H, I ; \Delta)$. Then $B_{\gamma} / \mathscr{H} \in \mathcal{U} \cap \mathscr{B} \subseteq \mathscr{V} \cap \mathscr{B} \subseteq \mathscr{V}$ and $H \in \mathscr{U} \cap \mathscr{G} \subseteq \mathscr{V} \cap \mathscr{G} \subseteq \mathscr{V}$. But $B_{\gamma}$ is isomorphic to the 0 -direct product $H \times_{0}\left(B_{\gamma} / \mathscr{H}\right)$. Therefore $B_{\gamma}$ is also in $\mathscr{V}$. Consequently, $S \in \mathscr{V}$ and $\mathcal{U} \subseteq \mathcal{V}$ if and only if $\mathcal{U} \psi \leq \mathscr{V} \psi$. To prove that $\psi$ is a lattice isomorphism it will suffice to show that it is an epimorphism.

Let $(\mathcal{U}, \mathscr{V}, \mathcal{W}) \in \mathscr{L}(\mathscr{R} \mathscr{B}) \times \mathscr{L}(\mathscr{B}) \times \mathscr{L}(\mathscr{G})$. Clearly $(\mathcal{U} \vee \mathscr{V} \vee \mathcal{W}) \psi \geq(\mathcal{U}, \mathscr{V}, \mathcal{W})$. To establish equality, it suffices to provide identities satisfied by $\mu=\mathscr{U} \vee \mathscr{V} \vee W$ which imply that $\mathcal{M} \cap \mathscr{R} \mathscr{B} \subseteq \mathcal{U}, \mathcal{M} \cap \mathscr{B} \subseteq \mathcal{V}$ and $\mathcal{M} \cap \mathscr{G} \subseteq \mathcal{W}$. It will follow that the identities given constitute a basis for $\mathcal{M}$ and also that every element of $\mathscr{L}\left(\mathscr{S}^{*}\right)$ is of the form $\mathcal{M}=\mathscr{U} \vee \mathcal{V} \vee W$ for $(\mathscr{U}, \mathscr{V}, W) \in \mathscr{L}(\mathscr{R} \mathscr{B}) \times \mathscr{L}(\mathscr{B}) \times \mathscr{L}(\mathscr{G})$. 
We shall only consider one representative case in detail and then provide a table of identities for the other cases.

Let $\mathcal{U}=\mathscr{L} \mathscr{Z}, \mathcal{V}=\mathscr{B}$ and let $\mathcal{W}$ be a group variety. By Lemma 3.2 , the variety of strict ${ }^{*}$-semigroups given by the identity $x x^{*}=y y^{*}$ is clearly the variety $\mathscr{G}$ of groups and consequently the group variety $W$ has a basis of identities of the form $x x^{*}=y y^{*}$ and $u_{\alpha x}^{2}=u_{\alpha}(\alpha \in A)$.

Consider the set of identities

$$
\text { (i) } x x^{*} y y^{*} z z^{*}=x x^{*} z z^{*} y y^{*}, \quad \text { (ii) } u_{\alpha}^{3}=u_{\alpha}^{2}(\alpha \in A) \text {. }
$$

These identities are clearly valid in $u, \mathcal{V}$ and $\mathcal{W}$. (Note that $x^{3}=x^{2}$ is an identity that holds in $\mathscr{B}$ from which (ii) follows.) Hence the identities (i) and (ii) are valid in $\boldsymbol{M}=\mathscr{U} \vee \mathscr{V} \vee \mathcal{W}$. However, any rectangular band satisfying (i) is necessarily a left zero semigroup so that $\mathscr{M} \cap \mathscr{R} \mathscr{B} \subseteq \mathscr{L} \mathscr{X}$, while any group satisfying (ii) must clearly lie in $W$ so that $\mathcal{M} \cap \mathscr{G} \subseteq W$. Trivially $\mathcal{M} \cap \mathscr{B} \subseteq \mathscr{B}=\mathscr{V}$ and so $\mathcal{M} \psi=(\mathscr{U}, \mathscr{V}, W)$ as required.

In Table 1 we tabulate suitable collections of identities for all possible cases involving a variety $\mathcal{W}$ of groups given by the identities $x x^{*}=y y^{*}, u_{\alpha}^{2}=u_{\alpha}(\alpha \in A)$. The first column gives $U \in \mathscr{L}(\mathscr{R} \mathscr{B})$ while the first row gives $\mathscr{V} \in \mathscr{L}(\mathscr{B})$ for the triple $(\mathcal{U}, \mathcal{V}, \boldsymbol{W})$. In every case we require the identities $u_{\alpha}^{3}=u_{\alpha}^{2}$ and the identities for strict *-semigroups, so we list only the additional identities required.

\begin{tabular}{|c|c|c|c|}
\hline & $\mathscr{T}$ & $\mathscr{\varphi}$ & $\mathscr{B}$ \\
\hline $\mathscr{T}$ & $x x^{*}=y y^{*}$ & $\begin{aligned} x x^{*} y y^{*} & =y y^{*} x x^{*} \\
x x^{*} & =x^{*} x\end{aligned}$ & $x x^{*} y y^{*}=y y^{*} x x^{*}$ \\
\hline $\mathscr{L E}$ & $x y y^{*}=x$ & $\begin{aligned} x y y^{*} z z^{*} & =x z z^{*} y y^{*} \\
x x^{*} & =x^{*} x\end{aligned}$ & $x y y^{*} z z^{*}=x z z^{*} y y^{*}$ \\
\hline $\mathscr{R} \mathscr{X}$ & $x x^{*} y=y$ & $\begin{aligned} x x^{*} y y^{*} z & =y y^{*} x x^{*} z \\
x x^{*} & =x^{*} x\end{aligned}$ & $x x^{*} y y^{*} z=y y^{*} x x^{*} z$ \\
\hline $\mathscr{R} \mathscr{B}$ & $x y y^{*} z=x z$ & $x x^{*}=x^{*} x$ & $\varnothing$ \\
\hline
\end{tabular}

Tahle 1

As remarked above, combining the identities in Table 1 with those for strict ${ }^{*}$-semigroups and $u_{\alpha}^{3}=u_{\alpha}^{2}(\alpha \in A)$ gives a basis for $\mathcal{U} \vee \mathscr{V} \vee \mathcal{W}$. Thus a basis for $\mathscr{R} \mathscr{Z} \vee \mathscr{B} \vee \mathcal{W}$ within $\mathscr{S}^{*}$ is $x x^{*} y y^{*} z=y y^{*} x x^{*} z, u_{\alpha}^{3}=u_{\alpha}^{2}$.

We illustrate our findings in Figure 1. In it, $\mathscr{S} \Phi$ denotes the variety of strict inverse semigroups and $\mathscr{C}$ stands for "combinatorial".

7. A structure theorem. In this section we develop a structure theorem for strict *-semigroups in terms of rectangular bands and inverse semigroups. The construction used is quite similar to that introduced by Yamada [16].

Let $T$ be a strict inverse semigroup. Since $\mathscr{D}=\mathscr{F}$ there is a natural partial ordering of the $\mathscr{D}$-classes of $T$. For each $\alpha \in T / \mathscr{D}$, let $B_{\alpha}$ be a rectangular band and, for $\beta \leq \alpha$, let $\theta_{\alpha, \beta}$ be a homomorphism of $B_{\alpha}$ into $B_{\beta}$ such that

(i) $\theta_{\alpha, \alpha}$ is the identity mapping on $B_{\alpha}$,

(ii) $\theta_{\alpha, \beta} \theta_{\beta, \gamma}=\theta_{\alpha, \gamma}(\gamma \leq \beta \leq \alpha)$. 


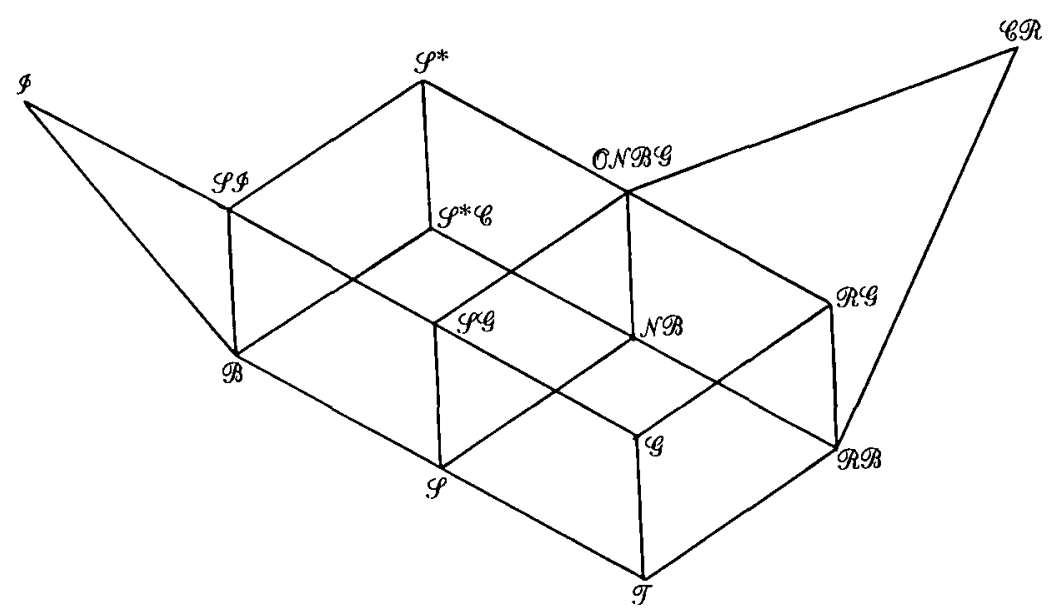

Figure 1

Let

$$
S\left(T ; B_{\alpha}, \theta_{\alpha, \beta}\right)=\left\{(e, a) \mid e \in B_{\alpha}, \quad \text { where } \quad \alpha=D_{a} \quad \text { and } \quad a \in T\right\},
$$

define a binary operation on $S\left(T ; B_{\alpha}, \theta_{\alpha, \beta}\right)$ by

$$
(e, a)(f, b)=\left(\left(e \theta_{\alpha, \gamma}\right)\left(f \theta_{\beta, \gamma}\right), a b\right)
$$

(where $\alpha=D_{a}, \beta=D_{b}, \gamma=D_{a b}$ ) and define a unary operation on $S\left(T ; B_{\alpha}, \theta_{\alpha, \beta}\right.$ ) by

$$
(e, a)^{*}=\left(e, a^{-1}\right) \text {. }
$$

THEOREM 7.1. With respect to the operations (3) and (4), $S\left(T ; B_{\alpha}, \theta_{\alpha, \beta}\right)$ is a strict ${ }^{*}$-semigroup and, conversely, every strict ${ }^{*}$-semigroup is *-isomorphic to one of this form.

Proof. The direct part of the proof follows from straightforward calculations. We consider just one of the many requirements to be verified, the proof of which is fairly typical. Consider identity (V). Let

$$
(e, a),(f, b),(g, c),(h, d) \in S\left(T ; B_{\alpha}, \theta_{\alpha, \beta}\right) .
$$

In order to simplify the notation, we write $\theta_{a, b}$ for $\theta_{D_{a} D_{b}}(a, b \in T)$. Then, writing $u=a c^{-1} b^{-1} d$, we have

$$
\begin{aligned}
(e, a)((f, b)(g, c))^{*}(h, d) & =(e, a)\left(\left(f \theta_{b, b c}\right)\left(g \theta_{c, b c}\right), b c\right)^{*}(h, d) \\
& =(e, a)\left(\left(f \theta_{b, b c}\right)\left(g \theta_{c, b c}\right), c^{-1} b^{-1}\right)(h, d) \\
& =\left(\left(e \theta_{a, u}\right)\left(f \theta_{b, u}\right)\left(g \theta_{c, u}\right)\left(h \theta_{d, u}\right), u\right)
\end{aligned}
$$

and similarly

$$
(e, a)(g, c)^{*}(f, b)^{*}(h, d)=\left(\left(e \theta_{a, u}\right)\left(g \theta_{c, u}\right)\left(f \theta_{b, u}\right)\left(h \theta_{d, u}\right), u\right) .
$$


The two first components here are equal, since in any rectangular band $x u v y=x y=x v u y$. Thus the two elements are equal and identity (V) holds.

Conversely, let $S$ be a strict *-semigroup. Let $\gamma$ be the minimum inverse semigroup congruence on $S$ and let $T=S / \gamma$. For $a \in S$ and $\alpha=D_{a}$, let $S_{\alpha}$ denote the principal factor $J(a) / I(a)$. For the sake of simplicity, we will denote the restriction of $\tau$ to any $\mathscr{D}$-class also by $\tau$. Let $I_{\alpha}=D_{a} / \tau$ (respectively, $\Lambda_{\alpha}=D_{a} / \tau^{\prime}$ ) be endowed with the structure of a left (respectively, right) zero semigroup and $B_{\alpha}$ denote the rectangular band $I_{\alpha} \times \Lambda_{\alpha}$.

For $\beta=D_{b}(b \in S)$ and $\beta \leq \alpha$, define $\theta_{\alpha, \beta}: B_{\alpha} \rightarrow B_{\beta}$ by

$$
\left(x \tau, y \tau^{\prime}\right) \theta_{\alpha, \beta}=\left(x \varphi_{\alpha, \beta} \tau, y \varphi_{\alpha, \beta} \tau^{\prime}\right)
$$

where $\varphi_{\alpha, \beta}$ is as in Notation 2.9. It follows from Lemmas 2.7 and 3.8 that $\theta_{\alpha, \beta}$ is well defined and it is then easily verified that $\theta_{\alpha, \beta}$ is a homomorphism. Since $\varphi_{\alpha, \alpha}$ is the identity mapping, so also is $\theta_{\alpha, \alpha}$ and, since $\varphi_{\alpha, \beta} \varphi_{\beta, \gamma}=\varphi_{\alpha, \gamma}$ when $\gamma \leq \beta \leq \alpha$ (Lemma 2.7(ii)), it also follows that $\theta_{\alpha, \beta} \theta_{\beta, \gamma}=\theta_{\alpha, \gamma}$. Thus we have all the necessary ingredients to construct $R=S\left(T ; B_{\alpha}, \theta_{\alpha, \beta}\right)$ and our next task is to show that $S$ is isomorphic to $R$.

Define $x: S \rightarrow R$ by

$$
x \chi=\left(\left(x \tau, x \tau^{\prime}\right), x \gamma\right) .
$$

From Lemma 4.6, we see that $\chi$ is one-to-one while, from Lemma 4.7, $\chi$ maps $S$ onto $R$.

To see that $\chi$ is a homomorphism, let $x, y \in S, \alpha=D_{x}, \beta=D_{y}$ and $\gamma=D_{x y}$. Then

$$
\begin{aligned}
(x \chi)(y \chi) & =\left(\left(x \tau, x \tau^{\prime}\right), x \gamma\right)\left(\left(y \tau, y \tau^{\prime}\right), y \gamma\right) \\
& =\left(\left(x \tau, x \tau^{\prime}\right) \theta_{\alpha, \gamma}\left(y \tau, y \tau^{\prime}\right) \theta_{\beta, \gamma},(x \gamma)(y \gamma)\right) \\
& =\left(\left(x \varphi_{\alpha, \gamma} \tau, x \varphi_{\alpha, \gamma} \tau^{\prime}\right)\left(y \varphi_{\beta, \gamma} \tau, y \varphi_{\beta, \gamma} \tau^{\prime}\right),(x y) \gamma\right) \\
& =\left(\left(x \varphi_{\alpha, \gamma} \tau, y \varphi_{\beta, \gamma} \tau^{\prime}\right),(x y) \gamma\right)
\end{aligned}
$$

while

$$
\left.(x y) \chi=\left((x y) \tau,(x y) \tau^{\prime}\right),(x y) \gamma\right) .
$$

Now $x y=\left(x \varphi_{\alpha, \gamma}\right)\left(y \varphi_{\beta, \gamma}\right) \Re x \varphi_{\alpha, \gamma}$ so that $(x y) \tau=x \varphi_{\alpha, \gamma} \tau$. Similarly, $(x y) \tau^{\prime}=y \varphi_{\beta, \gamma} \tau^{\prime}$ and the expressions (5) and (6) are equal. Thus $(x \chi)(y X)=(x y) \chi$ and the ${ }^{*}$-operation is clearly preserved.

8. An example. We conclude with an example to show that the *-operation on a strict *-semigroup need not be preserved under automorphisms. This also implies that a semigroup can be a strict *-semigroup with respect to two different unary operations.

Let $I=\{1\}, J=\{1,2\}$ and $S=(I \times J) \times{ }_{0} B_{2}$, where $I$ is a left zero semigroup and $J$ is a right zero semigroup. Then $S$ is a strict ${ }^{*}$-semigroup relative to the unary operation of Lemma 3.11. For $j \in J$ define

$$
\bar{j}=\left\{\begin{array}{lll}
2 & \text { if } & j=1 \\
1 & \text { if } & j=2
\end{array}\right.
$$


If $\theta: S \rightarrow S$ is defined by

$$
((1, j),(k, l)) \theta=\left\{\begin{array}{ll}
((1, j),(k, l)) & \text { if } \quad l=1, \\
((1, \bar{j}),(k, l)) & \text { if } \quad l=2,
\end{array} \text { and } 0 \theta=0,\right.
$$

then it is straightforward to see that $\theta$ is an automorphism of $S$. However,

$$
((1,1),(1,2))^{*} \theta=((1,1),(2,1)) \theta=((1,1),(2,1))
$$

while

$$
((1,1),(1,2)) \theta)^{*}=((1,2),(1,2))^{*}=((1,2),(2,1))
$$

so that $\boldsymbol{\theta}$ does not respect the ${ }^{*}$-operation. If we now define a unary operation $a \rightarrow a^{+}$on $S$ by

$$
a^{+}=\left((a \theta)^{*}\right) \theta^{-1}
$$

we obtain a unary operation on $S$ distinct from the ${ }^{*}$-operation and with respect to which $S$ is a strict ${ }^{*}$-semigroup, as is easily verified.

\section{REFERENCES} 434-451.

1. A. H. Clifford, The free completely regular semigroup on a set, J. Algebra 59 (1.979),

2. J. A. Gerhard, Free completely regular semigroups I, J. Algebra, to appear.

3. T. E. Hall and P. R. Jones, On the lattice of varieties of bands of groups, Pacific J. Math. 91 (1980), 327-337.

4. H.-J. Hoehnke, Über direkte Produkte vollständig einfacher Halbgruppen, Monatsb. Deutsch. Akad. Wiss. Berlin 4 (1962), 695-698.

5. J. M. Howie, An introduction to semigroup theory (Academic Press, 1976).

6. P. R. Jones, Completely simple semigroups: free products, free semigroups and varieties, Proc. Roy. Soc. Edinburgh Sect. A $\mathbf{8 8}$ (1981), 293-313.

7. E. I. Kleiman, On the lattice of varieties of inverse semigroups, Izv. Vyšs. Učebn. Zaved. Matematica 7 (1976), 106-109 (Russian). 209-218.

8. E. I. Kleiman, On bases of identities of Brandt semigroups, Semigroup Forum 13 (1977),

9. G. Lallement, Demi-groupes réguliers, Ann. Mat. Pura Appl. (4) 77 (1967), 47-129.

10. M. Petrich, Certain varieties and quasivarieties of completely regular semigroups, Canad. $J$. Math. 29 (1977), 1171-1197.

11. M. Petrich, Structure of regular semigroups (Cahiers Math. Montpellier, 1977).

12. N. R. Reilly, Varieties of completely semisimple inverse semigroups, J. Algebra 65 (1980), 427-444.

13. N. R. Reilly, Modular sublattices of the lattice of varieties of inverse semigroups, Pacific $J$. Math. 89 (1980), 405-517.

14. V. V. Rasin, On the lattice of varieties of completely simple semigroups, Semigroup Forum 17 (1979), 113-122.

15. V. V. Rasin, On the varieties of Cliffordian semigroups, Semigroup Forum 23 (1981), 201-220. 
16. M. Yamada, Regular semigroups whose idempotents satisfy permutation identities, Pacific J. Math. 21 (1967), 371-392.

17. M. Yamada, Generalized Brandt semigroups, Mem. Fac. Lit. Sci. Shimane Univ. Natur. Sci. No. 3 (1970), 1-8.

Simon Fraser UniverstTy, BURNABY, B.C.,

CANADA. 